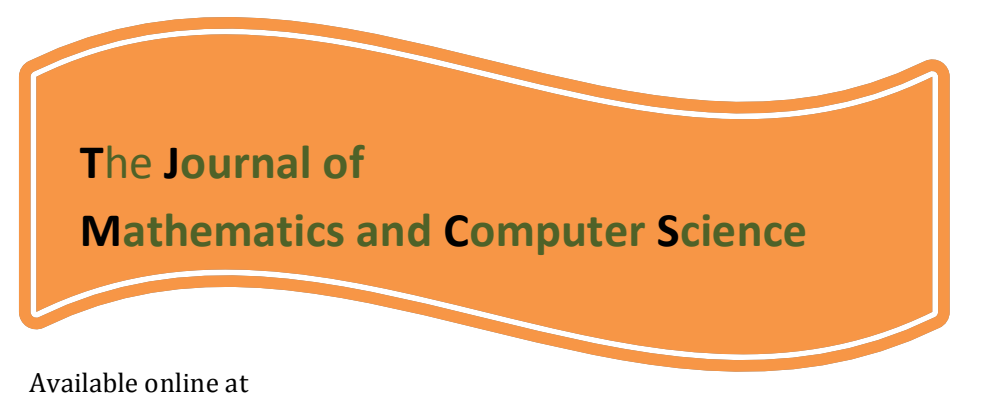

http://www.TJMCS.com

The Journal of Mathematics and Computer Science Vol. 4 No.1 (2012) 93 - 101

\title{
Random Numbers and Monte Carlo Approximation In Fuzzy Riemann Integral
}

\author{
Behuroz Fathi- Vajargah, Akram Heidary- Harzavily \\ Department of statistics, university of Guilan, Iran \\ fathi@guilan.ac.ir \\ heidary_math_88@yahoo.com
}

Received: December 2011, Revised: March 2012

Online Publication: May 2012

\begin{abstract}
In this paper, we want to improve Monte Carlo approximation in fuzzy Riemann integral it means that calculate exact amount of fuzzy Riemann integral based on $\alpha$ - level sets with partition of generating function of random numbers' RAND' in commercial software MATLAB.
\end{abstract}

Keywords: fuzzy Riemann integral, $\alpha$ - level sets.

\section{Introduction}

The fuzzy Riemann integral was introduced by $\mathrm{Wu}$ [2]. In Section 2, we give some basic properties of fuzzy sets and operations of fuzzy calculus [1], which will be used in the rest of this paper. In Section 3, we study on two kinds of fuzzy Riemann integrals. One is based on the crisp compact interval and the other one is considered on the fuzzy interval, where call them as fuzzy Riemann integral of type-I and type-II, respectively. Since the Riemann integral and Lebesgue integral are identical for a bounded function on a compact interval [2], we can apply the Lebesgue's monotone convergence theorem and dominated convergence Theorem to prove that the $\alpha$-level set of the fuzzy Riemann integral is a closed interval whose end points are the classical Riemann integrals. In Section $4, \mathrm{Wu}$ provided the computational methods and a number of examples to 
evaluate the fuzzy Riemann integrals numerically using the commercial software MATLAB.

In section 5, after using Monte Carlo approach to simulate of fuzzy Riemann integral, we express an example to use partition of generating function of random numbers' RAND' in MATLAB to reach exact amount of fuzzy Riemann integral.

\section{Basic definitions}

Let $(\mathrm{X}, \mathrm{M})$ be a measurable space and $(\mathrm{R}, \beta)$ be a Borel measurable space. Let $f: \mathrm{X} \rightarrow \mathrm{P}(\mathrm{R})$ (power set of $\mathrm{R}$ ) be a set-valued function. $f$ is called measurable if and only if $\{(x, y): \mathrm{y} \in$ $f(x)\}$ is $\mathrm{M} \times \beta$-measurable. If $\tilde{f}$ is a fuzzy-valued function then $\tilde{f_{\alpha}}$ is a set-valued function for all $\alpha \in[0,1] . \tilde{f}$ is called (fuzzy-valued) measurable if and only if $\tilde{f_{\alpha}}$ is (setvalued) measurable for all $\alpha \in[0,1]$.

Let $(\Omega, A, P)$ be a probability space (a complete $\sigma$-finite measure space).We know that $\mathrm{X}: \Omega \rightarrow \mathrm{R}$ is a random variable if $\mathrm{X}$ is an $(A, \beta)$-measurable function. Thus, we propose the following definition.

Definition 1. Let $\tilde{X}$ be a closed fuzzy-valued function. $\tilde{X}$ is called a fuzzy random variable if $\tilde{X}$ is measurable (or equivalently, strongly measurable).

Definition 2. Let $\tilde{X}$ be a closed fuzzy-valued function. $\tilde{X}$ is a fuzzy random variable if and only if $\tilde{X}_{\alpha}^{l}$ and $\tilde{X}_{\alpha}^{u} \alpha$ are random variables for all $\alpha \in[0,1]$.

Definition 3. Let $\tilde{X}$ and $\tilde{Y}$ be two fuzzy random variables. We say that $\tilde{X}$ and $\tilde{Y}$ are independent if each random variable in the $\operatorname{set}\left\{\tilde{X}_{\alpha}^{l}, \tilde{X}_{\alpha}^{u}: 0 \leq \alpha \leq 1\right.$ \}is independent of each random variable in the $\operatorname{set}\left\{\tilde{Y_{\alpha}^{l}}, \tilde{Y_{\alpha}^{u}}: 0 \leq \alpha \leq 1\right\}$. We say that $\tilde{X}$ and $\tilde{Y}$ are identically distributed if and only if $\tilde{X}_{\alpha}^{l}$ and $\tilde{Y}_{\alpha}^{l}$ are identically distributed and $\tilde{X}_{\alpha}^{u}$ and $\tilde{Y_{\alpha}^{u}}$ are identically distributed for all $\alpha \in[0,1]$.

Definition 4. Let $\tilde{X}$ be a fuzzy random variable. The (fuzzy) expectation of $\tilde{X}$ is defined by

$$
\tilde{E}(\tilde{X})=\int \tilde{X} d \tilde{x},(\tilde{E}(\tilde{X}))_{\alpha}=\left(\int \tilde{X} d \tilde{x}\right)_{\alpha}=\left[\int \tilde{X}_{\alpha}^{l} d p, \int \tilde{X}_{\alpha}^{u} d p\right]=\left[E\left(\tilde{X}_{\alpha}^{l}\right), E\left(\tilde{X}_{\alpha}^{u}\right)\right] .
$$

Definition 5. Let $\tilde{X}_{n}$ and $\tilde{X}$ be fuzzy random variables defined on the same probability $\operatorname{space}(\Omega, A, P)$. We say that $\tilde{X}_{n}$ converges to $\tilde{X}$ with probability one if $\left(\tilde{X}_{n}\right)_{\alpha}^{l}$ converges to $\tilde{X}_{\alpha}^{l} \alpha$ with probability one and $\left(\tilde{X}_{n}\right)_{\alpha}^{u}$ converges to $\tilde{X}_{\alpha}^{u}$ with probability one for all $\alpha \in[0,1]$.

Definition 6. We say that $\tilde{a}$ is a crisp number with value $\mathrm{m}$ if its membership function is

$$
\mu_{\tilde{a}}(r)=\left\{\begin{array}{cc}
1 & r=m \\
0 & \text { else }
\end{array}\right.
$$

$\tilde{a}$ is denoted by $\tilde{1}_{\{m\}}$. 
Theorem 1. (The Strong Law of Large Numbers for Fuzzy RandomVariables). Let be $\tilde{X}_{n}$ independent and identically distributed fuzzy random variables and $\sim \mu$ be the (fuzzy) expectation of $\tilde{X}_{n}$. Let $\tilde{Y}_{n}=\tilde{1}_{\left\{\frac{1}{n}\right\}} \tilde{x}\left(\tilde{X}_{1} \tilde{+} \tilde{X}_{2} \tilde{+} \ldots \tilde{+} \tilde{X}_{n}\right)$. Then $\tilde{Y}_{n}$ converges to $\mu$ with probability one [5].

Definition 7. Let $\mathrm{X}$ is a universal set. Then a fuzzy subset $\tilde{A}$ of $\mathrm{X}$ is defined by its membership function $\xi_{\tilde{A}}: \mathrm{X} \rightarrow[0,1]$. We can also write the fuzzy set $\tilde{A}$ as $\left\{\left(x, \xi_{\tilde{A}}(x)\right)\right.$ : $x \in \mathrm{X}\}$.We denote $\tilde{A_{\alpha}}=\left\{x: \xi_{\tilde{A}}(x) \geq \alpha\right\}$ as the $\alpha$ - level set $\tilde{A}$ of where $\tilde{A_{0}}$ is the closure of the $\operatorname{set}\left\{x: \xi_{\hat{A}}(x) \neq \alpha\right\}$ In this paper, we use $\mathrm{A}(x)$ as membership function of A instead of $\xi_{\tilde{A}}(x)$, just be comfortable.

Definition 8. A fuzzy set, $N$ from real numbers is called a real fuzzy number, if

(i) $\mathrm{N}$ is normal, it means that one and just one $x \in \mathrm{R}$ exsistes such that $\mathrm{N}(x)=1$.

(ii) $\alpha$ - level sets of $\mathrm{N}$ are closed intervals for $\alpha \in(0,1]$.

\section{Extension Principle of fuzzy numbers:}

We let $\tilde{M}$ and $\tilde{N}$ are two fuzzy numbers [5]. If $\tilde{*}$ denotes fuzzy addition, subtraction, multiplication, or division, then $\tilde{M} \tilde{*} \tilde{N}=\tilde{C}$, membership function of $\tilde{C}$ is defined $\tilde{C}(z)=\sup _{x, y}\{\operatorname{Min}(\tilde{M}(x), \tilde{N}(y)) \mid x * y=z\}$.

\section{Interval Arithmetic:}

We let $[a, b]$ and $[c, d]$ be two bounded, closed intervals of real numbers. If * denotes addition, subtraction, multiplication, or division, then

$$
[a, b]+[c, d]=[a+c, b+d],[a, b]-[c, d]=[a-d, c-b],
$$

If $*$ is divison, we must assume that zero does $\in$ not belong to $[c, d] .[a, b] /[c, d]=[a, b] \cdot\left[\frac{1}{d}, \frac{1}{c}\right]$ $[a, b] .[c, d]=[g, h]$ where $g=\min \{a c, a d, b d, b c\}$ and $h=\max \{a c, a d, b d, b c\}$.

Definition 9. Let $f(x)$ be a real-valued function on a topological space. If $\{x: f(x) \geq \alpha\}$ is closed for every $\alpha, f(x)$ is said to be upper semicontinuous [2].

Definition 10. (i) $\tilde{a}$ is called fuzzy number if $\tilde{a}$ is a normal convex fuzzy set and for all $\alpha$ the $\alpha$ - level set, $\tilde{a}_{\alpha}$, is bounded. The set of all fuzzy numbers is denoted as $\mathcal{F}$.

(ii) $\tilde{a}$ is called closed fuzzy number if $\tilde{a}$ is a fuzzy number and its membership function $\xi_{\tilde{a}}$ is upper semicontinuous. The set of all closed fuzzy numbers is denoted as $\mathcal{F}_{c l}$.

(iii) $\tilde{a}$ is called bounded fuzzy number if $\tilde{a}$ is a fuzzy number and its membership function $\xi_{\tilde{a}}$ has compact support. The set of all bounded fuzzy numbers is denoted as $\mathcal{F}_{b}$.

Proposition 1. If $\tilde{a}$ is a closed fuzzy number then the $\alpha$-level set of $\tilde{a}$ is a closed interval, which is denoted by $\tilde{a}_{\alpha}=\left[\tilde{a}_{\alpha}{ }^{L}, \tilde{a}_{\alpha}^{U}\right][2]$. 
Proposition 2. (i) Let $\tilde{A}$ be a fuzzy set with membership function $\xi_{\tilde{A}}$ and $A_{\alpha}=\left\{x: \xi_{\tilde{A}}(x) \geq \alpha\right\}$.Then

$$
\xi_{\tilde{A}}(x)=\sup _{0 \leq \alpha \leq 1} \alpha 1_{A_{\alpha}(x)}
$$

Where $1_{A_{\alpha}(x)}$, is membership function of $A_{\alpha}$ [8].

(ii) [9]Let $\mathrm{A}$ be a set and $\left\{A_{\alpha}: \alpha \in[0,1]\right\}$ be a family of subsets of $\mathrm{A}$ such that

(a) $A_{0}=A$,

(b) $A_{\alpha} \subseteq A_{\beta}$ for $\alpha<\beta$,

(c) $A_{\alpha}=\bigcap_{n=1}^{\infty} A_{\alpha_{n}}$ for $\alpha_{n} \uparrow \alpha$

Then the function $\xi: A \rightarrow[0,1]$ defined by

has the property that

$$
\xi(x)=\sup _{0 \leq \alpha \leq 1} \alpha 1_{A_{\alpha}(x)}
$$

$A_{\alpha}=\{x: \xi(x) \geq \alpha\}$ for all $\alpha \in[0,1]$.

Let $\left\{A_{\alpha}=\left[l_{\alpha}, u_{\alpha}\right]: 0 \leq \alpha \leq 1\right\}$ be a family of closed intervals. Then we can induce a fuzzy set $\widetilde{A}$ with membership function

$$
\xi_{\tilde{A}}(x)=\sup _{0 \leq \alpha \leq 1} \alpha 1_{A_{\alpha}(x)}
$$

We say that $\left\{A_{\alpha}\right\}$ is decreasing with respect to $\alpha$ if $A_{\beta} \subseteq A_{\alpha}$ for $\alpha<\beta[10]$.

Definition 11. Let $\mathcal{F}$ be a set of all fuzzy numbers, $\mathcal{F}_{c l}$ be a set of all closed fuzzy numbers and $\mathcal{F}_{b}$ be a set of all bounded fuzzy numbers. We say that

(i) $\quad \tilde{f}(x)$ is a fuzzy-valued function if $\tilde{f}: X \rightarrow \mathcal{F}$.

(ii) $\quad \tilde{f}(x)$ is a closed-fuzzy-valued function if $\tilde{f}: X \rightarrow \mathcal{F}_{c l}$

(iii) $\quad \tilde{f}(x)$ is a bounded-fuzzy-valued function if $\tilde{f}: X \rightarrow \mathcal{F}_{b}$

We denote $\tilde{f}_{\alpha}^{l}(x)=(\tilde{f}(x))_{\alpha}^{l}$ and $\tilde{f}_{\alpha}^{U}(x)=(\tilde{f}(x))_{\alpha}^{u}[2]$.

\section{3 . The Fuzzy Riemann Integral [2]}

Definition 12. Let $\tilde{f}(x)$ be a closed- and bounded-fuzzy-valued function on $[a, b]$. Suppose that for all $\alpha, \tilde{f}_{\alpha}^{L}(x)$ and $\tilde{f}_{\alpha}^{U}(x)$ are Riemann integrable on $[a, b]$. Let

$$
A_{\alpha}=\left[\int_{a}^{b} \tilde{f_{\alpha}^{l}}(x) d x, \int_{a}^{b} \tilde{f_{\alpha}^{U}}(x) d x\right]
$$

Then we say that $\tilde{f}(x)$ is fuzzy Riemann-integrable on $[a, b]$ with type-I, denoted as $\tilde{f}(x) \in \mathcal{F}_{R_{I}}$ on $[a, b]$, and the membership function of $\int_{a}^{b} \tilde{f}(x) d x$ is defined by, for $r \in A_{0}$,

$$
\mu_{\int_{a}^{b} \tilde{f}(x) d x}(x)=\sup _{0 \leq \alpha \leq 1} \alpha 1_{A_{\alpha}(x)}
$$


Given a real number $\mathrm{x}$, we can induce a fuzzy number $\tilde{x}$ with membership function $\xi_{\tilde{x}}(r)$ such that $\xi_{\tilde{x}}(r)=1$ and $\xi_{\tilde{x}}(r)<1$ for $\mathrm{r} \neq x$ (i.e. $\mathrm{r}=x$ is the unique global maximum of the membership function). We call $\tilde{x}$ as fuzzy real number induced by the real number $x$.

Let $\mathcal{F}_{R}$ be a set of all fuzzy real numbers induced by the real number system $\mathrm{R}$. We define the relation $\sim$ on $\mathcal{F}_{R}$ as $\tilde{x}^{1} \sim \tilde{x}^{2}$ if and only if $\tilde{x}^{1}$ and $\tilde{x}^{2}$ are induced by the same real number $\mathrm{x}$. Then $\sim$ is an equivalence relation inducing the equivalence classes $[\tilde{x}]=\{\tilde{a} \mid \tilde{a} \sim \tilde{x}\}$. The quotient set $\mathcal{F}_{R} / \sim$ is the set of all equivalence classes. Then the cardinality of $\mathcal{F}_{R} / \sim$ is equal to the cardinality of the real number system $\mathrm{R}$, since the map $R \rightarrow \mathcal{F}_{R} / \sim$ by $x \rightarrow[\tilde{x}]$ is a bijection. We call $\mathcal{F}_{R} / \sim$ as fuzzy real number system. In practice (for convenience), we take only one element $\tilde{x}$ from each equivalence class $[\tilde{x}]$ to form the fuzzy real number system $\left(\mathcal{F}_{R} / \sim\right)_{R}$ (roughly speaking), i.e. $\left(\mathcal{F}_{R} / \sim\right)_{R}=\{\tilde{x}$ । $\tilde{x} \in[\tilde{x}]$ is the only element from $[\tilde{x}]\}$.If the fuzzy real number system $\left(\mathcal{F}_{R} / \sim\right)_{R}$ consists of closed(bounded) fuzzy real numbers then we call $\left(\mathcal{F}_{R} / \sim\right)_{R}$ as closed (bounded) fuzzy real number system. Let $\tilde{f}:\left(\mathcal{F}_{R} / \sim\right)_{R} \rightarrow \mathcal{F}$ be a fuzzy-valued function defined on the fuzzy real number system $\left(\mathcal{F}_{R} / \sim\right)_{R}$ Suppose that $\tilde{x}$ is a fuzzy real number induced by a real number $x$. Then we can induce a new fuzzy-valued function $\tilde{f}: R \rightarrow \mathcal{F}$ by $\tilde{f}(x)=\tilde{f}(\tilde{x})$. Now, suppose that $\tilde{b}<\tilde{a}$, we shall discuss the fuzzy Riemann integrals on the fuzzy interval formed by $\tilde{a}$ and $\tilde{b}$ (denoted as $[\tilde{a}, \tilde{b}]$ ), where $\tilde{a}$ and $\tilde{b}$ are two closed fuzzy real numbers induced by two real numbers $a$ and $b$, respectively. In order to define the fuzzy Riemann integral of type-II, we need to consider the "length" between $\tilde{a}$ and $\tilde{b}$ for $\tilde{b}>\tilde{a}(\tilde{b} \geq \tilde{a})$ now $(\tilde{b}-\tilde{a})_{\alpha}^{l}=\tilde{b}_{\alpha}^{l}-\tilde{a}_{\alpha}^{u}$ and $(\tilde{b}-\tilde{a})_{\alpha}^{u}=\tilde{b}_{\alpha}^{u}-\tilde{a}_{\alpha}^{l}$. We shall consider the interval $\left[\tilde{a}_{\alpha}^{u}, \tilde{b}_{\alpha}^{l}\right]$ for the lower bound case and the interval $\left[\tilde{a}_{\alpha}^{l}, \tilde{b}_{\alpha}^{u}\right]$ for the upper bound case. Then we have the following definition.

Definition 13. Let $\tilde{f}(\tilde{x})$ be a bounded- and closed-fuzzy-valued function defined on the closed fuzzy real number $\operatorname{system}\left(\mathcal{F}_{R} / \sim\right)_{R}$, and $\tilde{\mathrm{f}}(x)$ be induced by $\tilde{f}(\tilde{x})$. Suppose that $\tilde{b}<\tilde{a}$.

(i) If $\tilde{f}(x)$ is nonnegative and $\tilde{f}_{\alpha}^{L}(x)$ and $\tilde{f}_{\alpha}^{U}(x)$ are Riemann-integrable on $\left[\tilde{a}_{\alpha}^{u}, \tilde{b}_{\alpha}^{l}\right]$ and $\left[\tilde{a}_{\alpha}^{l}, \tilde{b}_{\alpha}^{u}\right]$, respectively, for all $\alpha$ then we let

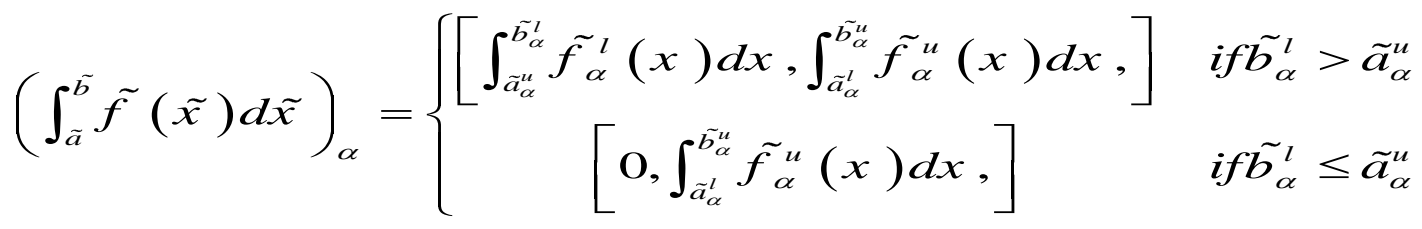

(ii) If $\tilde{f}(x)$ is nonpositive and $\tilde{f}_{\alpha}^{L}(x)$ and $\tilde{f}_{\alpha}^{U}(x)$ are Riemann-integrable on $\left[\tilde{a}_{\alpha}^{l}, \tilde{b}_{\alpha}^{u}\right]$ and $\left[\tilde{a}_{\alpha}^{u}, \tilde{b}_{\alpha}^{l}\right]$,respectively, for all $\alpha$ then we let 


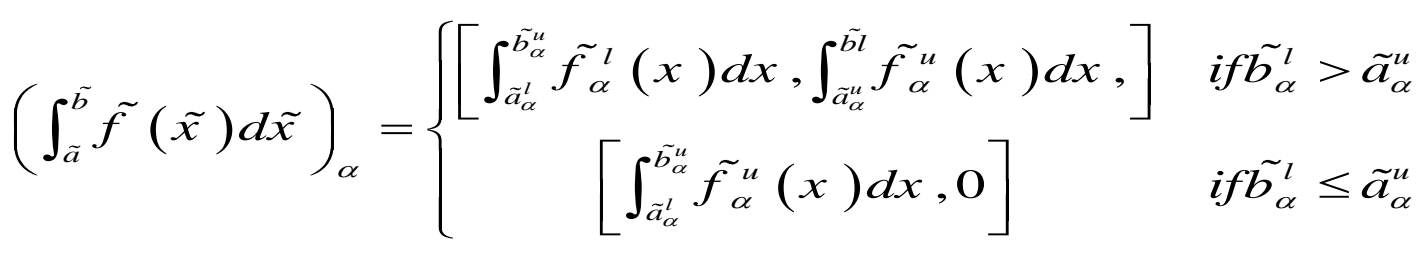

Under the above conditions, we say that $\tilde{f}(\tilde{x})$ is fuzzy Riemann-integrable on the fuzzy interval $[\tilde{a}, \tilde{b}]$ with type-II, denoted as $\tilde{f}(\tilde{x}) \in \mathcal{F}_{R_{I I}}$ on $[\tilde{a}, \tilde{b}]$, and the membership function of $\int_{\tilde{a}}^{\tilde{b}} \tilde{f}(\tilde{x}) d \tilde{x}$ is defined by, for $r \in A_{0}$,

$$
\mu_{\int_{\tilde{a}}^{\tilde{f}} \tilde{f}(x) d x}(x)=\sup _{0 \leq \alpha \leq 1} \alpha 1_{A_{\alpha}(x)} .
$$

\section{Computational methods}

Let $\mathrm{f}$ be a non fuzzy function from $\mathrm{X}$ to $\mathrm{Y}$ and $\tilde{x}$ be a fuzzy number. By the extension principle $[5,12]$, we can induce a fuzzy-valued function $\tilde{f}(\tilde{x})$ defined on the set of all fuzzy numbers. The membership function of $\tilde{f}(\tilde{x})$ is $\mu_{\tilde{f}(\tilde{x})}(r)=\sup _{r=f(y)} \mu_{\tilde{x}}(y)$.

Proposition 3. Let $f(\mathrm{x})$ be a real-valued function and $\tilde{f}(\tilde{x})$ be a fuzzy-valued function induced by $f(x)$ via the extension principle. Suppose that $\tilde{a}$ is a closed fuzzy number. If $\{x: r=f(x)\}$ is a compact set for all $\mathrm{r}$ then $(\tilde{f}(\tilde{a}))_{\alpha}=\left\{f(x): x \in \tilde{a}_{\alpha}\right\}$

Let $\{x: r=f(x)\}$ is compact set for all $r$.by the extension principle, we have

$$
\begin{gathered}
\tilde{f}_{\alpha}^{l}(x)=\inf _{y \in \tilde{x}_{\alpha}} f(y)=\min _{y \in \tilde{x}_{\alpha}} f(y) \\
\tilde{f}_{\alpha}^{u}(x)=\sup _{y \in \tilde{x}_{\alpha}} f(y)=\max _{y \in \tilde{x}_{\alpha}} f(y)
\end{gathered}
$$

Then

$$
\begin{aligned}
& \int_{a}^{b} \tilde{f_{\alpha}^{u}}(x) d x=\int_{a}^{b} \max _{y \in \tilde{x}_{\alpha}} f(y) d x \equiv g(\alpha) \\
& \int_{a}^{b} \tilde{f_{\alpha}^{l}}(x) d x=\int_{a}^{b} \min _{y \in \tilde{x}_{\alpha}} f(y) d x \equiv h(\alpha)
\end{aligned}
$$

Let $A_{\alpha}=[g(\alpha), h(\alpha)]$, membership function of $\int_{a}^{b} \tilde{f}(x) d x$ is

$$
\begin{gathered}
\mu(r)=\sup _{0 \leq \alpha \leq 1} 1_{A_{\alpha}}(r)=\max _{0 \leq \alpha \leq 1} 1_{A_{\alpha}}(r)=\max \left\{\alpha: 0 \leq \alpha \leq 1, r \in A_{\alpha}\right\} \\
\mu(r)=\max \alpha \\
g(\alpha) \leq r \leq h(\alpha) \\
\mathbf{O} \leq \boldsymbol{\alpha} \leq \mathbf{1}
\end{gathered}
$$

Now, we solve nonlinear programming[7].

\section{Monte Carlo approach to simulate of fuzzy Riemann integral}

Let $\mathrm{U}$ be a $\mathrm{U}(0,1)$ random variable. We set $\tilde{X}=\tilde{f}(U)$. Then $\tilde{X}_{\alpha}^{l}=\tilde{f}_{\alpha}^{l}(U)$ and $\tilde{X}_{\alpha}^{u}=\tilde{f_{\alpha}^{u}}(U)$ 
are random variables for all $\alpha \in[0,1]$ since $\tilde{f}_{\alpha}^{u}$ and $\tilde{f}_{\alpha}^{l}$ are measurable functions for all $\alpha$ $\in[0,1]$, we conclude that $\tilde{X}=\tilde{f}(U)$ is a fuzzy random variable .Let $\{$ Un\}be a sequence of independent $\mathrm{U}(0,1)$ random variables. From [6],we see that $\tilde{f}\left(U_{n}\right)$ are independent and identically distributed fuzzy random variables. From the strong law of large numbers for

random variables, we have

$$
\begin{aligned}
& \frac{1}{n}\left(\tilde{f_{\alpha}^{l}}\left(U_{1}\right)+\tilde{f_{\alpha}^{l}}\left(U_{2}\right)+\ldots+\tilde{f_{\alpha}^{l}}\left(U_{n}\right)\right) \rightarrow E\left(\tilde{f_{\alpha}^{l}}(U)\right)=\int_{0}^{1} \tilde{f_{\alpha}^{l}}(x) d x=\left(\int_{0}^{1} \tilde{f}(x) d x\right)_{\alpha}^{l} \\
& \frac{1}{n}\left(\tilde{f_{\alpha}^{u}}\left(U_{1}\right)+\tilde{f_{\alpha}^{u}}\left(U_{2}\right)+\ldots+\tilde{f_{\alpha}^{u}}\left(U_{n}\right)\right) \rightarrow E\left(\tilde{f_{\alpha}^{u}}(U)\right)=\int_{0}^{1} \tilde{f_{\alpha}^{u}}(x) d x=\left(\int_{0}^{1} \tilde{f}(x) d x\right)_{\alpha}^{u}
\end{aligned}
$$

with probability one. Therefore, from Theorem 1, we have

$$
\tilde{1}_{\left\{\frac{1}{n}\right\}} \tilde{x}\left(\tilde{f}\left(U_{1}\right) \tilde{+} \tilde{f}\left(U_{2}\right) \tilde{+} \ldots \tilde{+} \tilde{f}\left(U_{n}\right)\right) \rightarrow E(\tilde{f}(U))=\int_{0}^{1} \tilde{f}(x) d x .
$$

with probability one. Hence we can generate a large number of random numbers $\left\{U_{i}\right\}$ and take $\tilde{1}_{\left\{\frac{1}{n}\right\}} \tilde{\times}\left(\tilde{f}\left(U_{1}\right) \tilde{+} \tilde{f}\left(U_{2}\right) \tilde{+} \ldots \tilde{+} \tilde{f}\left(U_{n}\right)\right)$ as the approximation of the fuzzy Riemann integral $\int_{0}^{1} \tilde{f}(x) d x$.This approach to approximating the fuzzy Riemann integrals is called the Monte Carlo approach.(MC)

Let $f^{\sim}$ be a closed fuzzy-valued measurable function defined on $[a, b]$, and let $f$ be fuzzy Riemann-integrable on $[a, b]$. We consider to simulate with MC the fuzzy Riemann integral $\int_{a}^{b} \tilde{f}(x) d x$, we have $\left(\int_{a}^{b} \tilde{f}(x) d x\right)_{\alpha}=\left[\int_{a}^{b} \tilde{f_{\alpha}^{l}}(x) d x, \int_{a}^{b} \tilde{f_{\alpha}^{u}}(x) d x\right]$

$$
\int_{a}^{b} \tilde{f}_{\alpha}^{l}(x) d x=\int_{0}^{1} \tilde{g}_{\alpha}^{l}(x) d x \text { and } \int_{a}^{b} \tilde{f}_{\alpha}^{u}(x) d x=\int_{0}^{1} \tilde{g}_{\alpha}^{u}(x) d x
$$

That $\tilde{g}_{\alpha}^{l}(x)=(b-a) \tilde{f}_{\alpha}^{l}(a+(b-a) x)$ and $\tilde{g}_{\alpha}^{u}(x)=(b-a) \tilde{f}_{\alpha}^{u}(a+(b-a) x)$ From the strong law of large numbers for random variables, we have

$$
\begin{gathered}
\frac{b-a}{n}\left(\tilde{f_{\alpha}^{u}}\left(a+(b-a) U_{1}\right)+\ldots+\tilde{f_{\alpha}^{u}}\left(a+(b-a) U_{n}\right)\right) \rightarrow\left(\int_{a}^{b} \tilde{f}(x) d x\right)_{\alpha}^{u} \\
\frac{b-a}{n}\left(\tilde{f_{\alpha}^{l}}\left(a+(b-a) U_{1}\right)+\ldots+\tilde{f_{\alpha}^{l}}\left(a+(b-a) U_{n}\right)\right) \rightarrow\left(\int_{a}^{b} \tilde{f}(x) d x\right)_{\alpha}^{l} \\
1_{\left\{\frac{b-a}{n}\right\}} \tilde{\times}\left(\tilde{f}\left(a+(b-a) U_{1}\right) \tilde{+} \ldots \tilde{+} \tilde{f}\left(a+(b-a) U_{n}\right) \rightarrow \int_{a}^{b} \tilde{f}(x) d x .\right.
\end{gathered}
$$

Let $f^{\sim}$ be a closed fuzzy-valued measurable function defined on $[a, b]$, and let $f^{\sim}$ be fuzzy Riemann-integrable on $[a, \infty]$. We consider to simulate with MC the fuzzy Riemann integral

$$
\begin{aligned}
& \int_{a}^{\infty} \tilde{f_{\alpha}^{u}}(x) d x=\int_{0}^{1} \tilde{g}_{\alpha}^{u}(x) d x \text { and } \int_{a}^{\infty} \tilde{f_{\alpha}^{l}}(x) d x=\int_{0}^{1} \tilde{g}_{\alpha}^{l}(x) d x \text { then } \\
& \tilde{g}_{\alpha}^{l}(x)=\frac{\tilde{f_{\alpha}^{l}}\left(\frac{1}{x}+(a-1)\right)}{x^{2}} \text { and } \tilde{g}_{\alpha}^{u}(x)=\frac{\tilde{f_{\alpha}^{u}}\left(\frac{1}{x}+(a-1)\right)}{x^{2}} \\
& \frac{1}{n}\left(\frac{\tilde{f_{\alpha}^{u}}\left(\frac{1}{U_{1}}+(a-1)\right)}{U_{1}^{2}}+\ldots+\frac{\tilde{f_{\alpha}^{u}}\left(\frac{1}{U_{n}}+(a-1)\right)}{U_{n}{ }^{2}}\right) \rightarrow\left(\int_{a}^{\infty} \tilde{f}(x) d x\right)_{\alpha}^{u}
\end{aligned}
$$


$\frac{1}{n}\left(\frac{\tilde{f_{\alpha}^{l}}\left(\frac{1}{U_{1}}+(a-1)\right)}{U_{1^{2}}^{2}}+\ldots+\frac{\tilde{f_{\alpha}^{l}}\left(\frac{1}{U_{n}}+(a-1)\right)}{U_{n}^{2}}\right) \rightarrow\left(\int_{a}^{\infty} \tilde{f}(x) d x\right)_{\alpha}^{l}$ with probability one.

$\left.\tilde{1}_{\left\{\frac{1}{n}\right\}} \tilde{x}\left(\tilde{1}_{\left\{\frac{1}{U_{1}^{2}}\right\}} \tilde{\times} \tilde{f}\left(\left(\frac{1}{U_{1}}\right)+(a-1)\right)\right) \tilde{+} \ldots \tilde{+}\left(\tilde{1}_{\left\{\frac{1}{U_{n}^{2}}\right\}} \tilde{x} \tilde{f}\left(\frac{1}{U_{n}}\right)+(a-1)\right)\right) \rightarrow \int_{a}^{\infty} \tilde{f}(x) d x$

with

probability one. Hence we can generate a large number of random numbers $\left\{U_{i}\right\}$ and take

$\left.\left.\tilde{1}_{\left\{\frac{1}{n}\right\}} \tilde{x}\left(\tilde{1}_{\left\{\frac{1}{U_{1}^{2}}\right\}} \tilde{x} \tilde{f}\left(\frac{1}{U_{1}}\right)+(a-1)\right)\right) \tilde{+} \ldots \tilde{+}\left(\tilde{1}_{\left\{\frac{1}{U_{n}^{2}}\right\}} \tilde{x} \tilde{f}\left(\frac{1}{U_{n}}\right)+(a-1)\right)\right)$ as the approximation of the improper fuzzy Riemann integral $\int_{a}^{\infty} \tilde{f}(x) d x$.

The improper fuzzy Riemann integrals $\int_{-\infty}^{b} \tilde{f}(x) d x$ and $\int_{-\infty}^{\infty} \tilde{f}(x) d x$ can also be evaluated similarly using the Monte Carlo method.

Let $\tilde{f}(x)$ be a closed and bounded fuzzy-valued function on $[a, b]$. Suppose that $\tilde{f_{\alpha}^{l}}$ and $\tilde{f_{\alpha}^{u}}$

are continuous on $[a, b]$ for all $\alpha \in[0,1]$; then, we have generated a sequence of random numbers $\left\{U_{i}\right\}$.Let

$$
\begin{gathered}
A_{\alpha}=\left[\frac { b - a } { n } \left(\tilde{f_{\alpha}^{l}}\left(a+(b-a) U_{1}\right)+\ldots+\tilde{f}_{\alpha}^{l}\left(a+(b-a) U_{n}\right), \frac{b-a}{n}\left(\tilde{f_{\alpha}^{u}}\left(a+(b-a) U_{1}\right)+\ldots+\tilde{f}_{\alpha}^{u}\left(a+(b-a) U_{n}\right)\right] \equiv[g(\alpha), h(\alpha)]\right.\right. \\
\tilde{y}_{n}(a, b)=\tilde{1}_{\left\{\frac{b-a}{n}\right\}} \tilde{\times}\left(\tilde{f}\left(a+(b-a) U_{1}\right) \tilde{+} \ldots \tilde{+} \tilde{f}\left(a+(b-a) U_{n}\right)\right) \\
\mu_{\tilde{y}_{n}(a, b)}=\sup _{0 \leq \alpha \leq 1} \alpha \cdot 1_{A_{\alpha}}=\max \alpha \\
g(\alpha) \leq r \leq h(\alpha) \\
\mathbf{O} \leq \alpha \leq \mathbf{1}
\end{gathered}
$$

\section{Example}

Let $\tilde{f}(x)=(\tilde{x} \tilde{x} \tilde{x}) \tilde{+}(\tilde{2} \tilde{x} \tilde{x}) \tilde{+} \tilde{1}$ be a closed fuzzy-valued function, where $\tilde{x}=(x-1, x, x+$ 1)

Is positive triangular fuzzy numbers [1] and $x \geq 1$. Then we want to evaluate the fuzzy Riemann integral $\int_{1}^{3} \tilde{f}(x) d x$. First of all, according to fuzzy arithmetic, we have

$$
\tilde{f_{\alpha}^{l}}(x)=2 \alpha^{2}+\alpha(3 x-1)+\left(x^{2}-x\right)
$$

And

$$
\tilde{f}_{\alpha}^{u}(x)=2 \alpha^{2}-\alpha(3 x+7)+\left(x^{2}+5 x+6\right)
$$

with MC approach[6],

$g(\alpha) \approx 4 \alpha^{2}+10 \alpha+\frac{14}{3}$ and $h(\alpha) \approx 4 \alpha^{2}-26 \alpha$, we have

$h(1)=g(1) \approx \frac{56}{3}=\int_{1}^{3}\left(x^{2}+2 x+1\right) d x$

By solving methods of system [6],we have down table. 
Behuroz Fathi- Vajargah, Akram Heidary- Harzavily/ TJMCS Vol. 4 No. 1 (2012) 93 - 101

\begin{tabular}{|c|c|}
\hline $\mathrm{r}$ & \\
\hline 15.000 & 0.786131 \\
\hline 16.000 & 0.846624 \\
\hline 17.000 & 0.905419 \\
\hline 18.000 & 0.962653 \\
\hline 18.666 & 1.000000 \\
\hline 19.000 & 0.981557 \\
\hline 20.000 & 0.927106 \\
\hline 21.000 & 0.873903 \\
\hline
\end{tabular}

Then, the fuzzy Riemann integral is about $\frac{56}{3}$.

Instead of using of up computing, in (1-2),we applied partition of generating function of random numbers' RAND' in MATLAB we take exactly $\left(\frac{56}{3}\right)$ amount of this integral.

\section{Reference}

[1] H.J. Zimmermann, theory and its applications, 2nd Edition, kluwer Academic, Boston, 1991.

[2] H.-C. Wu, The fuzzy Riemann integral and its numerical integration, Fuzzy set and System 110(2000), 1-25

[3] T. M. Apostol, Mathematical Analysis, 2nd ed., Addison-Wesly, Reading, MA, 1974.

[4] J. Buckly, Fuzzy probability and statistics ("studies in Fuzziness and Soft Computing") Springer (2006).

[5] H.-C. $\mathrm{Wu}$, The improper fuzzy Riemann integral and its numerical, integration, Inform. .Sci. 11 (1999), 109-137.

[6] H.-C.Wu, Evaluate Fuzzy Riemann Integrals Using the Monte Carlo Method, Journal of Mathematical Analysis and Applications 264,324-343 (2001).

[7] M.S. Bazarra, C.M Shetty, Nonlinear programming, Wiley,New York, 1993.

[8] W. Rudin, Real and Complex Analysis, McGraw-Hill, New York, 1986.

[9] M. Sakawa, H. Yano, Feasibility and pareto optimality for multiobjective linear and linear fractional programming problems with fuzzy parameters, in: J.L. Verdegay, M. Delgado (Eds.), The Interface between Arti_cial Intelligence and Operations Research in Fuzzy Environment, Verlag TUV Rheinland, 1990, pp. (213-232).

[10] J.R. Sims, Z.Y. Wang, Fuzzy measures and fuzzy integrals: an overview, Internat. J. Gen. Systems 17 (1990) (157-189). 